\title{
386.
}

\section{ON THE LOGARITHMS OF IMAGINARY QUANTITIES.}

[From the Proceedings of the London Mathematical Society, vol. II. (1866-1869), pp. 50—54. Read Dec. 12, 1867.]

THE theory of the logarithms of imaginary quantities admits of a remarkably simple representation.

Let $P$ denote at pleasure the imaginary quantity $x+i y$, or else the point the coordinates of which are $(x, y)$; viz., $P$ regarded as a quantity will denote $x+i y$; but we may also speak of the point $P$.

Writing thus

and similarly

we have of course

$$
\begin{aligned}
& P=x+i y, \\
& P^{\prime}=x^{\prime}+i y^{\prime},
\end{aligned}
$$

$$
\frac{P}{P^{\prime}}=\frac{x+i y}{x^{\prime}+i y^{\prime}},
$$

an imaginary quantity $X+i Y$; and the point $\frac{P}{P^{\prime}}$ will be the point the coordinates of which are $(X, Y)$.

We have

$$
P=r e^{i \theta},
$$

viz., $r$ is $=\sqrt{x^{2}+y^{2}}$, the radical being positive, and $\theta$ is an are such that

$$
\cos \theta=\frac{x}{\sqrt{x^{2}+y^{2}}}, \quad \sin \theta=\frac{y}{\sqrt{x^{2}+y^{2}}},
$$

and moreover $\theta$ may be taken to be an arc between the limits $-\pi,+\pi$. The are so defined may be denoted by $\tan ^{-1} \frac{y}{x}$, so that we have $\theta=\tan ^{-1} \frac{y}{x}$. 
It is to be observed that $\theta$ has always a determinate unique value, except in the single case $y=0, x$ negative, where we have indeterminately $\theta= \pm \pi$.

It is further to be remarked that, taking $A$ for the origin of coordinates, we have $\theta=$ angle $x A P$, considered as positive or as negative according as $P$ lies above or below the axis of $x$.

Starting from the equation

we have similarly

and

$$
\begin{aligned}
& P=r e^{i \theta}, \\
& P^{\prime}=r^{\prime} e^{i \theta^{\prime},} \\
& \frac{P}{P^{\prime}}=\frac{r}{r^{\prime}} e^{i \phi},
\end{aligned}
$$

where $\phi$ is derived from $\frac{P}{P^{\prime}}$ in the same way as $\theta$ from $P$, or $\theta^{\prime}$ from $P^{\prime}$.

Consequently

$$
e^{i\left(\theta-\theta^{\prime}-\phi\right)}=1,
$$

and therefore $\theta-\theta^{\prime}-\phi$ a multiple of $2 \pi$, say

$$
\theta-\theta^{\prime}-\phi=2 m \pi
$$

and in this equation the value of $m$ is determined by the limiting conditions above imposed on the values of $\theta, \theta^{\prime}, \phi$. To see how this is, suppose in the first instance that the finite line or chord $P^{\prime} P$, considered as drawn from $P^{\prime}$ to $P$, cuts the negative part of the axis of $x$ upwards; $P$ is then above, $P^{\prime}$ below, the axis of $x$; that is, $\theta,-\theta^{\prime}$ are each positive; and drawing the figure, it at once appears that the sum $\theta+\left(-\theta^{\prime}\right)$, that is $\theta-\theta^{\prime}$, is a positive quantity greater than $\pi$. And in this case the angle $\phi$ will be equal to $2 \pi-\left(\theta-\theta^{\prime}\right)$ taken negatively, that is, $\phi=-\left\{2 \pi-\left(\theta-\theta^{\prime}\right)\right\}$, or $\theta-\theta^{\prime}-\phi=2 \pi$. But, in like manner, if $P^{\prime} P$ cut the negative part of the axis of $x$ downwards, $P$ will be below, $P^{\prime}$ above, the axis of $x ;-\theta$ and $\theta^{\prime}$ are here each positive, and the figure shows that the sum $-\theta+\theta^{\prime}$ is greater than $\pi$; and in this case the angle $\phi$ is $=2 \pi-\left(-\theta+\theta^{\prime}\right)$; that is, we have $\theta-\theta^{\prime}-\phi=-2 \pi$. In every other case, (that is, if the chord $P^{\prime} P$ either does not meet the axis of $x$, or if it meets the positive part of the axis of $x, \theta-\theta^{\prime}$ and $\phi$ are each in absolute magnitude less than $\pi$, and we have $\theta-\theta^{\prime}-\phi=0$. So that we see that, according as the chord $P^{\prime} P$, considered as drawn from $P^{\prime}$ to $P$, meets the negative part of the axis of $x$ upwards or downwards, or as it does not meet the negative part of the axis of $x$, the value of $\theta-\theta^{\prime}-\phi$ is $=2 \pi,=-2 \pi$, or $=0$.

Taking now $\log r$ to represent the real logarithm of the positive real quantity $r$, we may, as a definition of the logarithm of the imaginary quantity $P(=x+i y)$, write

$$
\log P=\log r+i \theta
$$

The value so defined is of course one out of the infinite number of values of the logarithm, and it may for distinction be termed the "selected" value. In all that follows, the symbol " $\log$ " is to be understood to denote the selected value. We have

$$
\log P=\log r+i \theta
$$


and similarly

and

$$
\log P^{\prime}=\log r^{\prime}+i \theta^{\prime}
$$

$$
\log \frac{P}{P^{\prime}}=\log \frac{r}{r^{\prime}}+i \phi
$$

Hence

$$
\log P-\log P^{\prime}=\log \frac{P}{P^{\prime}}+i\left(\theta-\theta^{\prime}-\phi\right)
$$

so that, by what precedes, $\log P-\log P^{\prime}$, if the chord $P^{\prime} P$, considered as drawn from $P^{\prime}$ to $P$, cuts the negative part of the axis of $x$ upwards, is $=\log \frac{P}{P^{\prime}}+2 i \pi$; if the chord cuts the negative part of the axis of $x$ downwards, it is $=\log \frac{P}{P^{\prime}}-2 i \pi$, and in every other case it is $=\log \frac{P}{P}$.

It is to be remarked that $\log P$, as above defined, is a continuous function of $P(=x+i y)$, with the single exception that, if the point $P$ move from above to below or from below to above the negative part of the axis of $x$, the imaginary part of the logarithm changes from $+i \pi$ to $-i \pi$, or from $-i \pi$ to $+i \pi$, in the two cases respectively. And we are thus led to another mode of looking at the question.

Consider the integral

$$
\int_{P^{\prime}}^{P} \frac{d z}{z}
$$

The value of the integral may depend on the series of values assumed by the variable $z$ as it passes from the limit $z=P^{\prime}$ to the limit $z=P$, or say it may depend on the path of the variable $z$; in order to give the notation a precise signification, we must therefore fix the path of the variable $z$; and I do this by taking the path to be the right line $P^{\prime} P$. Write now $z=P^{\prime}$. u, we have $\frac{d z}{z}=\frac{d u}{u} ; z=P^{\prime}$ gives $u=1 ; z=P$ gives $u=\frac{P}{P^{\prime}}$; and it is easy to see that, the path of $z$ being along the right line $P^{\prime}$ to $P$, that of $u$ is along the right line 1 to $\frac{P}{P^{\prime}}$ (that is, from the point the coordinates whereof are $x=1, y=0$, to the point $\left.\frac{P}{P^{\prime}}\right)$.

We have thus

$$
\int_{P^{\prime}}^{P} \frac{d z}{z}=\int_{1}^{P \div P^{\prime}} \frac{d u}{u}
$$

the path in each case being a right line as above. The indefinite integral $\int \frac{d u}{u}=\log u$; and as $u$ passes from 1 to $\frac{P}{P^{\prime}}$, there is no discontinuity in the value of $\log u$; the 
value of the right-hand side is thus $=\log \frac{P}{P^{\prime}}$. As regards the left-hand side, the indefinite integral is in like manner $=\log z$; but here, if the chord $P^{\prime} P$ cuts the negative part of the axis of $x$, there is a discontinuity in the value of $\log z$, viz., if the chord $P^{\prime} P$, considered as drawn from $P^{\prime}$ to $P$, cuts the negative part of the axis of $x$ upwards, there is an abrupt change in the value of $\log z$ from $-i \pi$ to $+i \pi$; and similarly, if the chord cut the negative part of the axis of $x$ downwards, there is an abrupt change from $+i \pi$ to $-i \pi$; in the former case, by taking the definite integral to be $\log P-\log P^{\prime}$, we take its value too large by $2 i \pi$, in the latter case we take it too small by $2 i \pi$; that is, the true value of the definite integral is in the former case $=\log P-\log P^{\prime}-2 i \pi$, in the latter case it is $=\log P-\log P^{\prime}+2 i \pi$. But if the chord $P P^{\prime}$ does not cut the negative part of the axis of $x$, then there is not any discontinuity, and the true value of the definite integral is $=\log P-\log P^{\prime}$. We have thus in the three cases respectively

$$
\begin{aligned}
\log P-\log P^{\prime} & =\log \frac{P}{P^{\prime}}+2 i \pi \\
& =\log \frac{P}{P^{\prime}}-2 i \pi \\
& =\log \frac{P}{P^{\prime}}
\end{aligned}
$$

which agrees with the previous results.

It may be remarked, that it is merely in consequence of the particular definition adopted that there is in the value of $\log P$ a discontinuity at the passage over the negative part of the axis of $x$; with a different definition of the logarithm, there would be a discontinuity at the passage over some other line from the origin; but a discontinuity somewhere there must be. For if, as above, the chord $P^{\prime} P$ meet the negative part of the axis of $x$, then forming a closed quadrilateral by joining by right lines the points 1 to $P, P$ to $P^{\prime}, P^{\prime}$ to $\frac{P}{P^{\prime}}$, and $\frac{P}{P^{\prime}}$ to 1 ; the only side meeting the negative part of the axis of $x$ is the side $P^{\prime} P$; the integral $\int \frac{d z}{z}$, taken through the closed circuit in question, or say the integral

$$
\left(\int_{P}^{1}+\int_{P^{\prime}}^{P}+\int_{P \div P^{\prime}}^{P^{\prime}}+\int_{1}^{P \div P^{\prime}}\right) \frac{d z}{z}
$$

has, by what precedes, a value in consequence of the discontinuity in passing from $P^{\prime}$ to $P$; viz., this is $=-2 i \pi$ or $=2 i \pi$, according as the chord $P^{\prime} P$, considered as drawn from $P^{\prime}$ to $P$, cuts the negative part of the axis of $x$ upwards or downwards; but this value $-2 i \pi$ or $+2 i \pi$ must be altogether independent of the definition of the logarithm; whereas if, by any alteration in the definition, the discontinuity could be avoided, the value of the integral, instead of being as above, would be $=0$. The foregoing value $-2 i \pi$ or $+2 i \pi$ is in fact that of the integral taken along (in the one c. VI. 
or the other direction) any closed curve surrounding the point $z=0$ for which the function $\frac{1}{z}$ under the integral sign becomes infinite: but in obtaining the value as above, no use is made of the principles relating to the integration of functions which thus become infinite.

The equation

gives

$$
\log P=\log r+i \theta
$$

$$
P^{m}=e^{m \log P}=r^{m} e^{i m \theta},
$$

or say

$$
(x+i y)^{n}=r^{m} e^{i m \theta},
$$

where, $m$ being any real quantity whatever, $r^{m}$ denotes the positive real value of $r^{m}$. We have thus a definition of the value of $(x+i y)^{m}$, and the value so defined may be called the selected value. And similarly, for an imaginary exponent $m=p+q i$, we have

$$
\begin{aligned}
(x+i y)^{p+q i} & =e^{(p+q i)(\log r+i \theta)} \\
& =e^{p \log r-q \theta+i(p \theta+q \log r)} \\
& =r^{p} e^{-q \theta} \cdot e^{i(p \theta+q \log r)},
\end{aligned}
$$

which is the selected value of $(x+i y)^{p+q i}$.

It may be remarked, in illustration of the advantage (or rather the necessity) of having a selected value, that in an integral $\int Z d z$, taken between given limits along a given path, it is necessary that we know, for the real or imaginary value of $z$ corresponding to each point of the path, the value of the function $Z$; and consequently, if $Z$ is a function involving $\log z$ or $z^{m}$, the indeterminateness which presents itself in these symbols (considered as belonging to a single value of $z$ ) is, so to speak, indefinitely multiplied, and $\int Z d z$ is really an unmeaning combination of symbols, unless by selecting, as above or otherwise, a unique value of $\log z$ or $z^{m}$, we render the function to be integrated a determinate function of the variable. 